\title{
LOS BUPRÉSTIDOS DEL CUADRANTE NOROCCIDENTAL ESPAÑOL (COLEOPTERA, BUPRESTIDAE)
}

\author{
L. Arnáiz Ruiz (*)
}

\begin{abstract}
RESUMEN
En el presente trabajo se realiza un estudio faunístico de la familia Buprestidae Leach, 1815 en el cuadrante noroccidental español; abarcando las siguientes provincias: La Coruña, Lugo, Orense, Pontevedra, Asturias, Cantabria, Vizcaya, Guipúzcoa, Álava, Navarra, La Rioja, Soria, Burgos, Palencia, León, Valladolid, Zamora y Salamanca. Se incluyen nuevos datos de captura de 79 táxones (sp. y ssp.), primeras citas para varias provincias españolas. Con los datos nuevos aportados y la revisión de datos bibliográficos se actualiza el conocimiento de la distribución de 103 táxones. El listado de las especies se presenta indicando localidades por provincias y referencias bibliográficas donde se ha citado cada especie. Además, se realizan correcciones a la nomenclatura en algunos de los táxones tratados en este estudio.
\end{abstract}

Palabras clave: Coleoptera, Buprestidae, corología, nomenclatura, cuadrante noroccidental de España.

\section{ABSTRACT \\ The buprestid beetles from Northwest Spain (Coleoptera, Buprestidae)}

In this paper a faunistic study of species of family Buprestidae Leach, 1815 from north-western part of Spain has been realized, including the following provinces: La Coruña, Lugo, Orense, Pontevedra, Asturias, Cantabria, Vizcaya, Guipúzcoa, Álava, Navarra, La Rioja, Soria, Burgos, Palencia, León, Valladolid, Zamora y Salamanca. New data of 79 taxa (sp. and ssp.), recorded for the first time from several spanish provinces, are included. With the bibliographic and new data brought out, the knowledge about distribution of 103 taxa are updated. A check-list of species containing the provinces, new localities and bibliographic references where the species are cited, is given. Moreover, corrections to the nomenclature of some taxa studied in the present work are done.

Key words: Coleoptera, Buprestidae, corology, nomenclature, north-western part of Spain.

\section{Introducción}

Recientemente se han publicado diversos trabajos que contribuyen al conocimiento matizado de la distribucíón peninsular de algunos bupréstidos y en concreto en la zona de estudio (Arnáiz Ruiz, 1996a y 1997).

En la excelente monografía de la familia Buprestidae del Dr. A. Cobos (1986), recientemente fallecido, queda patente que el conocimien-

* C/ Ferrer n ${ }^{\circ}$ 23. Vegas del Condado 24153 (León). 
to de la corología de esta familia en el cuadrante noroccidental español es fragmentario, echándose en falta datos que concreten la distribución real de la mayoría de las especies pertenecientes a esta familia en ese área peninsular. En este contexto, el presente trabajo aporta una serie de datos corológicos nuevos que, junto con los datos bibliográficos ya conocidos, actualizan y detallan la distribución de mas de un centenar de especies pertenecientes a esta familia en el área de estudio (Apéndice 1).

\section{Material y métodos}

El material que ha servido de base para la elaboración del presente estudio faunistico procede de las siguientes fuentes:

- Colección del Dr. P. Bahillo de la Puebla (Barakaldo-Vizcaya) (col. PB).

- Colecciones de: D. I. Alonso, D. J. Alcorta, D. R. Fernández de Gamboa y S.C.N.A. (Sociedad de Ciencias Naturales Aranzadi-San Sebastian). Datos comunicados al autor por el Dr. P. Bahillo de la Puebla. (col. IA) (col. JA) (col. RFG) y (col. SCNA).

- Colección de D. M. A. Sánchez Sobrino (Madrid) (col. MS).

- Colección de D. J. L. Agoiz Bustamante (Tudela-Navarra) (col. JLA).

- Colección de D. J. I. Lopéz-Colón (Rivas Vaciamadrid-Madrid) (col. JLC).

- Colección de D. F. Calvo (Renteria-Guipúzcoa) (col. FC).

- Colección de D. R. Martínez Fernández (Montejos-León) (col. RM).

- Colección P. Bercedo - L. Arnáiz (Vegas del Condado-León) (col. BA).

Se debe tener presente que esta aportación no es en modo alguno definitiva y que, con toda probabilidad, el presente catálogo se verá incrementado con nuevos táxones y/o con nuevas localizaciones de los táxones aquí citados.

\section{Resultados}

Subfamilia BUPRESTINAE Leach, 1815

Tribu ACMAEODERINI Kerremans, 1893

Género Acmaeodera Eschscholtz, 1829

Subgénero Acmaeodera Eschscholtz, 1829

1.- Acmaeodera (Acmaeodera) pilosellae (Bonelli, 1812)

SORIA: Pobar (Teunissen, 1998).
LEON: Ambasaguas de Curueño, 7-VI-98, L. Arnáiz leg. (col. BA)

SALAMANCA: Puebla de Azaba, 30-III-97, F. Calvo leg. (col. FC).

\section{2.- Acmaeodera (Acmaeodera) pulchra}

(Fabricius, 1792)

LA RIOJA: Logroño (Cobos, 1986).

Subgénero Acmaeotethya Volkovitsh, 1979

3.- Acmaeodera (Acmaeotethya) crinita Gory, 1840 ssp. melanosoma Lucas, 1844

NAVARRA: Ramalete (Tudela), 31-VI-94, J. Agoiz leg. (col. BA) y Las Norias (Tudela), 12-VI-97, J. Agoiz leg. (col. JLA).

SALAMANCA: Izcala, 25-VI-85, T. Martínez leg. (col. $\mathrm{RM})$.

4.- Acmaeodera (Acmaeotethya) degener (Scopoli, 1763) ssp. quattuordecimpunctata (Villers, 1789)

ÁLAVA: Huetos, 7-VII-90, I. Alonso leg. (col. PB) $\mathrm{BA})$

CANTABRIA: Cambarco, 2-VIII-95, L. Arnáiz leg. (col.

LEÓN: Valdeón, 16-VII-85, R. Martínez leg. (col. RM).

SALAMANCA: Puebla de Azaba, 20-VI-97, F. Calvo leg. (col. FC).

NAVARRA: Iregua, 19-VII- 81, I. Alonso leg. (col. IA).

Subgénero Palaeotethya Volkovitsh, 1979 5.- Acmaeodera (Palaeotethya) bipunctata (Olivier, 1790)

PALENCIA: Bárcena de Campos, 29-VII-96, P. Bahillo leg. (col. PB).

LEÓN: Vegas del Condado, 17-VI-98, L. Arnáiz leg. (col. BA) y Ambasaguas de Curueño, 7-VI-98, L. Arnáiz leg. (col. BA).

SALAMANCA: La Alberca, 11-VI-94, M. Sobrino leg. (col. MS).

\section{6.- Acmaeodera (Palaeotethya) nigellata Abeille,} 1904

PONTEVEDRA: Alto del Páramo (debe tratarse de Alto del Paraño, en la provincia de Orense) (Cobos, 1986).

LEÓN: Guiana, Mts. Aquilinos, El Teleno, La Barreira, Villarino (Cobos, 1986).

Género Acmaeoderella Cobos, 1955 Subgénero Acmaeoderella Cobos, 1955

7.- Acmaeoderella (Acmaeoderella) moroderi (Reitter, 1906)

LEÓN: Pedrún, 17-VI-84, R. Martínez leg. (col. RM).

Subgénero Carininota Volkovitsh, 1979 8.-Acmaeoderella (Carininota) flavofasciata (Piller \& Mitterpacher, 1783) ssp. flavofasciata

ÁLAVA: Huetos, 7-VII-90, 21-VII-91, I. Alonso leg. (col. IA); Laguardia, 11-VII-90, I. Alonso leg. (col. IA), 25-VI-91, 
J. Alcorta leg. (col. JA); Pto. Herrera, 11-VII-90, I. Alonso leg. (col. IA); Leza, 11-VII-92, P. Bahillo leg. (col. PB); Sarria, 23 VII-92, P. Bahillo leg. (col. PB) y Urbasa, 25-VII-78, (col. SCNA)

CANTABRIA: Potes, 13-VI-80, P. Bercedo leg. (col. BA) y Cosgaya, 17-VII-86, L. Arnáiz leg. (col. BA).

NAVARRA: Azuelo, 20-VI-93, P. Bahillo leg. (col. PB) y Biguezal, 20-VI-98, F. Calvo leg. (col. FC).

BURGOS: Oña, 21-VII-91, L. Arnáiz leg. (col. BA).

PALENCIA: Pto. de Piedras Luengas, 11-VIII-89, P.

Bahillo leg. (col. PB).

SORIA: Adradas, 25-VII-97, L. Arnáiz leg. (col. BA) y

Cuevas de Agreda, 26-VI-98, M. Sobrino leg. (col. MS)

LEÓN: Riaño, 4-VI-85, R. Martínez leg. (col. RM); Inicio,

28-VI-84, R. Martínez leg. (col. RM); Irede, 19-VIII-84, R.

Martínez leg. (col. RM); Barrios de Luna, 7-VII-94, L. Arnáiz

leg. (col. BA); Lugán, 16-VI-98, L. Arnáiz leg. (col. BA) y

Vegas del Condado, 16-VII-98, L. Arnáiz leg. (col. BA).

ORENSE: Carballino (Cobos, 1986).

PONTEVEDRA: Aldea de Los Quiñós, 12-VIII-81,

López-Colón leg. (col. JLC).

\section{9.- Acmaeoderella (Carininota) flavofasciata}

(Piller \& Mitterpacher, 1783) ssp. pilivestis (Abeille, 1904)

LEÓN: Truchas (Cobos, 1986); Valderas, 7-VII-84, R. Martínez leg. (col. RM); Bosque de Horma (Riaño), 3-VII-84, R. Martínez leg. (col. RM) y León alrededores, 16-VI-84, R. Martínez leg. (col. RM).

ZAMORA: Ribadelago (Cobos, 1986)

SALAMANCA: La Alberca (Cobos, 1986) y Sa de Béjar (Cobos, 1986).

Subgénero Euacmaeoderella Volkovitsh, 1979

10.- Acmaeoderella (Euacmaeoderella) cyanipennis (Lucas, [1846]) ssp. hispana (Abeille, 1900)

SORIA: Tardajos de Duero (Teunissen, 1998) y Adradas, 25-VII-97, L. Arnáiz leg. (col. BA). BA).

LEON: Vegas del Condado, 11-VII-98, L. Arnáiz leg. (col.

SALAMANCA: San Martín de Yelpes (debe tratarse de Martín de Yeltes) (Teunissen, 1998).

\section{1.- Acmaeoderella (Euacmaeoderella)} lanuginosa (Gyllenhal, 1817)

ZAMORA: Granucillo de Vidriales, 11-VII-93, P. Bahillo leg. (col. PB).

LEÓN: Valderas, 7-VII-84, 26-VIII-84, R. Martínez leg. (col. RM).

Subgénero Omphalothorax Volkovitsh, 1979 12.- Acmaeoderella (Omphalothorax) adspersula (Illiger, 1803)

PALENCIA: Bárcena de Campos, 2-VIII-97, P. Bahillo leg. (col. PB).

ORENSE: Razamonde, 18-VII-90, M. Sobrino leg. (col. MS).

SALAMANCA: Puebla de Azaba, 25-VIII-97, 10-IX-97, F. Calvo leg. (col. FC).
BA).

LEÓN: Vegas del Condado, 4-VIII-98, L. Arnáiz leg. (col.

Tribu POLYCESTINI Lacordaire, 1857

Género Ptosima Solier, 1833

13.- Ptosima flavoguttata (Illiger, 1803)

ASTURIAS: Mt. Muniellos, Las Brañas (Cobos, 1986).

LEÓN: León alrededores, 8-VI-79, U. Abella leg. (col. RM).

ORENSE: Razamonde, 15-VI-93, M. Sobrino leg. (col. MS).

Tribu CHALCOPHORINI Lacordaire, 1857

Género Chalcophora Solier, 1833

14.- Chalcophora mariana (Linnaeus, 1758) ssp. massiliensis (Villers, 1789)

BURGOS: Oña, 21-VII-91, L. Arnáiz leg. (col. BA) y Medina de Pomar, 22-VI-85, P. Bahillo leg. (col. PB).

SORIA: Garray, 1-VI-90, L. Arnáiz leg. (col. BA) y Navaleno, 11-VIII-80, J. R. Beltrán leg. (col. JLC).

VALLADOLID: Ataquines, 2-XI-78, P. Bercedo leg. (col. BA).

SALAMANCA: Puebla de Azaba, 22-VIII-95, F. Calvo leg. (col. FC).

Género Capnodis Eschscholtz, 1829

15.- Capnodis tenebrionis (Linnaeus, 1761)

VIZCAYA: Bazán, 1-X-96, R. F. de Gamboa leg. (col. RFG).

PALENCIA: Bárcena de Campos, 3-VIII-95, P. Bahillo leg. (col. PB).

16.- Capnodis tenebricosa (Olivier, 1790)

NAVARRA: Mendavia (sin fecha), J. Agoiz leg. (col. JLA).

Género Cyphosoma Mannerheim, 1837

17.- Cyphosoma lausoniae (Chevrolat, 1838)

ZAMORA: Villatófila (debe tratarse de Villafáfila) (Cobos, 1986).

Tribu BUPRESTINI Leach, 1815

Género Dicerca Eschscholtz, 1829

Subgénero Dicerca Eschscholtz, 1829

18.- Dicerca (Dicerca) berolinensis (Herbst, 1779)

LA RIOJA: Logroño (Cobos, 1986).

Género Poecilonota Eschscholtz, 1829

19.- Poecilonota variolosa (Paykull, 1799) ssp. variolosa

LA RIOJA: Logroño (Cobos, 1986)

Género Palmar Schaefer, 1949

20.- Palmar festiva (Linnaeus, 1767)

SORIA: Bocigas de Perales (Cobos, 1986).

Género Eurythyrea Lacordaire, 1835

21.- Eurythyrea quercus (Herbst, 1780)

VIZCAYA: Cortézubi (Cobos, 1986); Butrón, 10-VIII-92, I. Alonso leg. (col. IA); El Regato, 16-VII-89, I. Alonso leg. (col. IA) y Usabel, 13-VIII-92, I. Alonso leg. (col. PB). 
GUIPÚZCOA: Sa de Aralar (Cobos, 1986).

ASTURIAS: Mt. Muniellos, Las Brañas (Cobos, 1986).

CANTABRIA: Maoño, 6-IX-79, P. Bercedo leg. (col. BA).

Género Buprestis Linnaeus, 1758

Subgénero Buprestis Linnaeus, 1758

22.- Buprestis (Buprestis) haemorrhoidalis Herbst, 1780

CANTABRIA: Ruanales, 1-VII-81, P. Bercedo leg. (col. BA).

\section{3.- Buprestis (Buprestis) novemmaculata} Linnaeus, 1767

VIZCAYA: Ochandio, 11-VIII-91, J. Alcorta leg. (col. JA); Rioseco, 17-VII-88, J. Alcorta leg. (col. JA); Urigoiti, 113-IX87, J. Alcorta leg. (col. JA); Carranza (Lanzas Agudas), 20VIII-91, I. Alonso leg. (col. IA); Usabel, 13-VIII-92, I. Alonso leg. (col. IA) e Itxina, 29-VIII-98, I. Alonso leg. (col. PB).

ÁLAVA: Barrio, 9-IX-94, P. Bahillo leg. (col. PB); Nograro, 12-IX-92, P. Bahillo leg. (col. PB); Pto. CrucetaOchandio, 17-IX-89, P. Bahillo leg. (col. PB); Villafranca de San Zadornil, 12-IX-92, P. Bahillo leg. (col. PB); Zaldibartzo, 11-VIII-95, R. F. de Gamboa leg. (col. RFG) y Valderejo, 5VIII-92, I. Alonso leg. (col. IA).

NAVARRA: Garde, 30-V-97, L. Arnáiz leg. (col. BA).

LA RIOJA: Villoslada de Cameros, 21-VIII-93, I. Alonso leg. (col. IA).

BURGOS: Oña, 21-VII-91, L. Arnáiz leg. (col. BA).

PALENCIA: Bárcena de Campos, 10-VIII-89, P. Bahillo leg. (col. PB).

VALLADOLID: Villalba de los Alcores, 19-VII-97, L. S. Sánchez leg. (col. FC).

SORIA: Pto. de Santa Inés, 24-VII-93, J. Alcorta leg. (col. JA); Abejar, 6-VIII-78, P. Bercedo leg. (col. BA) y Garray, 1VI-90, L. Arnáiz leg. (col. BA).

SALAMANCA: La Alberca, 1-VII-93, M. Sobrino leg. (col. MS).

ORENSE: Razamonde, 23-VII-90, M. Sobrino leg. (col. MS) y Leiro, 19-VII-90, M. Sobrino leg. (col. MS).

LEÓN: Vegas del Condado, 18-VII-98, 30-VIII-98, L. Arnáiz leg. (col. BA) y Valderas, 5-V-84, Rosa Ma Castañeda leg. (col. RM).

24.- Buprestis (Buprestis) octoguttata Linnaeus, 1758 ÁLAVA: Barrio, 9-IX-94, P. Bahillo leg. (col. PB) y Valderejo, 9-XII-92, I. Alonso leg. (col. BA).

SORIA: Pto. de Santa Inés, 10-VIII-94, P. Bahillo leg. (col PB) y 18-VIII-94, I. Alonso leg. (col. BA).

25.- Buprestis (Buprestis) rustica Linnaeus, 1758 NAVARRA: Garde, 30-V-97, L. Arnáiz leg. (col. BA). SORIA: Pto. de Santa Inés, 18-VIII-94, I. Alonso leg. (col. Bellamy).

Género Anthaxia Eschscholtz, 1829

Subgénero Cratomerus Solier, 1833

26.- Anthaxia (Cratomerus) hungarica (Scopoli, 1772)

ÁLAVA: Buxanda, 20-VIII-93, P. Bahillo leg. (col. PB); Huetos, 6-VI-92, P. Bahillo leg. (col. PB); Laguardia, 11-VII-
90, I. Alonso leg. (col. IA); Pobes, 14-V-94, P. Bahillo leg. (col. PB) y $\mathrm{S}^{\mathrm{a}}$ de Arkamo-Osma, 2-VII-95, R. F. de Gamboa leg. (col. RFG).

LEÓN: Villaceid, 24-VI-84, R. Martínez leg. (col. RM); Sena de Luna, 1-VI-84, R. Martínez leg. (col. RM); Remellán, 23-VI-84, R. Martínez leg. (col. RM); Bosque de Horma (Riaño), 3-VII-84, R. Martínez leg. (col. RM); Ambasaguas de Curueño, 6-VI-98, 11-VII-98, L. Arnáiz leg. (col. BA); Santa María del Condado, 29-V-98, L. Arnáiz leg. (col. BA); Jiménez de Jamuz, 8-VIII-92, P. Bahillo leg. (col. PB).

NAVARRA: Meano, 9-VII-92, J. Alcorta leg. (col. JA) y San Cristobal (Pamplona), 25-VII-90, I. Alonso leg. (col. IA). LA RIOJA: Torremontalbo, 25-V- 96, J. A. Soto leg. (col. RFG).

PALENCIA: Bárcena de campos, 8-VII-89, 4-VII-98, P. Bahillo leg. (col. PB) (col. BA).

BURGOS: Valdenoceda, 2-V-87, I. Alonso leg. (col. IA). SORIA: Adradas, 25-VII-97, L. Arnáiz leg. (col. BA).

ZAMORA: Granucillo de Vidriales, 29-VI-93, P. Bahillo leg. (col. PB).

SALAMANCA: Puebla de Azaba, 27-IV-96, 1-XI-96, 7XII-96, 30-III-97, F. Calvo leg. (col. FC).

Subgénero Haplanthaxia Reitter, 1911 27.- Anthaxia (Haplanthaxia) millefolii

(Fabricius, 1801) ssp. polychloros Abeille, 1894 ÁLAVA: Huetos, 1-VII-91, P. Bahillo leg. (col. PB); Laguardia, 3-VII-91, J. Alcorta leg. (col. JA); Sarria, 18-VIII91, P. Bahillo leg. (col. PB) y Gopegui, 7-VII-94, P. Bahillo leg. (col. PB).

NAVARRA: Biguezal, 20-VI-98, F. Calvo leg. (col. FC).

LA RIOJA: Ledesma de la Cogolla, 8-VII-92, J. Alcorta

leg. (col. JA).

PALENCIA: Bárcena de Campos, 13-VIII-93, 4-VII-98, P. Bahillo leg. (col. PB) (col. BA).

LEÓN: Pedrún, 17-VI-84, R. Martínez leg. (col. RM); Olleros, 20-VI-98, L. Arnáiz leg. (col. BA) y Santovenia de la Valdoncina, 7-VII-94, L. Arnáiz leg. (col. BA).

ORENSE: Razamonde, 20-VII-90, M. Sobrino leg. (col. MS).

BURGOS: Olmillos de Sasamón, 15-VI- 97, M. Sobrino leg. (col. MS).

ZAMORA: Cervantes, VII-81, M. A. López Prada leg. (col. JLC) y Granucillo de Vidriales, 8-VII-93, P. Bahillo leg. (col. PB).

SALAMANCA: Puebla de Azaba, 27-IV-97, 15-VII-97, F. Calvo leg. (col. FC) y Sequeros, 29-VI-95, M. Tomás leg. (col. $\mathrm{FC})$.

28.- Anthaxia (Haplanthaxia) ignipennis Abeille, 1882

LA RIOJA: Nieva de Cameros (Cobos, 1986).

SALAMANCA: Béjar (Cobos, 1986).

\section{9.- Anthaxia (Haplanthaxia) parallela Laporte \& Gory, 1839}

VALLADOLID: Iscar, 12-IV-97, P. Bahillo leg. (col. PB).

SALAMANCA: El Payo, 4-VII-83, A. Mateos leg. (col. JLC); Peñaparda, 25-VI-83, 26-VII-83, H. Santos leg. (col. JLC) y Robleda, 2-VII-83, A. Justo leg. (col. JLC). 
LEÓN: Ambasaguas de Curueño, 7-VI-98, L. Arnáiz leg. (col. BA).

30.- Anthaxia (Haplanthaxia) scutellaris Gené, 1839

VIZCAYA: Galdames, 28-VI-92, I. Alonso leg. (col. IA) (col. PB).

\section{1.- Anthaxia (Haplanthaxia) umbellatarum} (Fabricius, 1787)

ORENSE: Razamonde, 18-VII-90, 25-VII-90, M. Sobrino leg. (col. MS) (col. BA).

Subgénero Anthaxia Eschscholtz, 1829

32.- Anthaxia (Anthaxia) mendizabali Cobos, 1965

LEÓN: Ambasaguas de Curueño, 28-VI-98, L. Arnáiz leg. (col. BA).

33.- Anthaxia (Anthaxia) manca (Linnaeus, 1767) ÁLAVA: Contrasta, 9-X-93, P. Bahillo leg. (col. PB).

BURGOS: Bujedo (debe tratarse de Bugedo-Orón) (Cobos, 1986) $\mathrm{RM})$.

LEÓN: Puente Castro, 6-V-71, F. Vega Escandón leg. (col.

ORENSE: Razamonde, 16-IV-89, M. Sobrino leg. (col. MS)

\section{4.- Anthaxia (Anthaxia) hypomelaena (Illiger,} 1803)

ÁLAVA: Gopegui, 7-VII-94, P. Bahillo leg. (col. PB) y Huetos, 6-VI-92, P. Bahillo leg. (col. PB).

NAVARRA: Tudela, 20-V-96, J. Agoiz leg. (col. JLA) y Viana, 2-VI-94, 11-VI-94, P. Bahillo leg. (col. PB) (col. BA).

LA RIOJA: San Vicente de Sonsierra, 4-VII-92, P. Bahillo leg. (col. PB)

SORIA: Borobia, $\mathrm{S}^{\mathrm{a}}$ del Moncayo, 19-VIII-77, Von Helversen leg. (col. H. Mühle). BA)

LEÓN: Vegas del Condado, 7-VII-98, L. Arnáiz leg. (col.

35.- Anthaxia (Anthaxia) funerula (Illiger, 1803) ssp. funerula

NAVARRA: Soto de Ramalete (Tudela), 22-IV-94, J. Agoiz leg. (col. JLA).

CANTABRIA: Cueto (Santander), 14-III-77, P. Bercedo leg. (col. BA).

PALENCIA: Pto. de Piedras Luengas, 11-VIII-89, P. Bahillo leg. (col. PB) y Cervera de Pisuerga, 5-IX-96, L. Arnáiz leg. (col. BA).

LEÓN: Ambasaguas de Curueño, 7-VI-98, L. Arnáiz leg. (col. BA).

\section{6.- Anthaxia (Anthaxia) fulgurans (Schrank, 1789) \\ NAVARRA: Biguezal, 20-VI-98, F. Calvo leg. (col. FC).}

37.- Anthaxia (Anthaxia) thalassophila Abeille, 1900 ssp. iberica Cobos, 1986

NAVARRA: Biguezal, 20-VI-98, F. Calvo leg. (col. FC); Soto de la Remonta (Tudela), 28-V-97, 6-VI-97, J. Agoiz leg. (col. JLA) y Chopera de Ramalete (Tudela), 28-V-97, 6-VI-97, J. Agoiz leg. (col. JLA) (col. BA). BA)

SORIA: Hinojosa de la Sierra, 4-VII-93, J. Agoiz leg. (col.

38.- Anthaxia (Anthaxia) salicis (Fabricius, 1777)

ÁLAVA: S ${ }^{a}$ de Urbasa, 30-VI-96, 24-V-97, F. Calvo leg. (col. FC).

NAVARRA: Garde, 29-V-97, L. Arnáiz leg. (col. BA)

SALAMANCA: La Alberca, 11-VI-94, M. Sobrino leg. (col. MS)

LEÓN: Luyego, 6-V-98, L. Arnáiz leg, (col. BA) y Vegas del Condado, 26-IV-98, L. Arnáiz leg. (col. BA)

39.- Anthaxia (Anthaxia) nitidula (Linnaeus, 1758)

ÁLAVA: $S^{a}$ de Urbasa, 7-VI-97, 10-V-98, F. Calvo leg. (col. FC).

GUIPÚZCOA: Oñate (Cobos, 1986).

BURGOS: Burgos alrededores (Cobos, 1986).

LEÓN: Valdeón, 6-VII-85, T. Martínez leg. (col. RM); Adrados, 21-VI-98, L. Arnáiz leg. (col. BA) y Oville, 19-VI98, L. Arnáiz leg. (col. BA).

40.- Anthaxia (Anthaxia) semicuprea Küster, 1851 ÁLAVA: Sa de Urbasa, 24-V-97, F. Calvo leg. (col. FC).

NAVARRA: Garde, 29-V-97, L. Arnáiz leg. (col. BA) y Pto. de Arteta-Goñi, 31-V-97, J. Agoiz leg. (col. JLA).

41.- Anthaxia (Anthaxia) dimidiata (Thunberg, 1789) SALAMANCA: Puebla de Azaba, 20-VI-97, F. Calvo leg. (col. FC).

42.- Anthaxia (Anthaxia) cyanescens Gory, 1841 ssp. cyanescens

VALLADOLID: Valladolid alrededores (Cobos, 1986).

LEÓN: Villafelíz de la Sobarriba, 18-IV-98, L. Arnáiz leg. (col. BA).

43.- Anthaxia (Anthaxia) cyanescens Gory, 1841 ssp. bedeli Abeille, 1893

PALENCIA: Bárcena de Campos, 22-IV-92, P. Bahillo leg. (col. PB).

ZAMORA: Granucillo de Vidriales, 17-IV-92, 28-III-97, P. Bahillo leg. (col. PB)

Subgénero Melanthaxia Richter, 1949 44.- Anthaxia (Melanthaxia) godeti Laporte \& Gory, 1839

ÁLAVA: Lalastra, 12-IX-92, P. Bahillo leg. (col. PB) y Huetos, 5-V-96, R. F. de Gamboa leg. (col. RFG).

GUIPÚZCOA: Oyarzun, 23-VI-91, F. Calvo leg. (col. FC) y Rentería, 16-VI-98, F. Calvo leg. (col. FC).

VIZCAYA: Ochandio, 11-VIII-91, J. Alcorta leg. (col. JA).

NAVARRA: Garde, 29-V-97, L. Arnáiz leg. (col. BA).

SORIA: Ctra. de Valvanera, 7-VII-91, J. Alcorta leg. (col. JA) y Pto. de Piqueras, 16-VI-98, I. Zabalegui leg. (col. FC).

LEÓN: Candanedo de Fenar, 20-VI-98, L. Arnáiz leg. (col. BA) y Santa María del Condado, 23-VI-98, L. Arnáiz leg. (col. BA). 
PALENCIA: Bárcena de Campos, 25-VII-97, 4-VII-98, P. Bahillo leg. (col. PB) (col. BA).

\section{5.- Anthaxia (Melanthaxia) sepulchralis}

(Fabricius, 1801)

ÁLAVA: Barrio, 9-IX-94, P. Bahillo leg. (col. PB) y Laguardia (sin fecha), J. Alcorta leg. (col. JA).

NAVARRA: Garde, 29-V-97, L. Arnáiz leg. (col. BA); Isaba, 30-V-97, L. Arnáiz leg. (col. BA) y Alto de Erro, 31-V97, L. Arnáiz leg. (col. BA).

PALENCIA: Bárcena de Campos, 18-III-94, P. Bahillo leg. (col. BA).

LEÓN: Vegas del Condado, 26-IV-98, 18-V-98, 20-V-98, 10-VI-98, 13-VI-98, L. Arnáiz leg. (col. BA) y Ambasaguas de Curueño, 7-VI-98, L. Arnáiz leg. (col. BA)

46.- Anthaxia (Melanthaxia) nigrojubata Roubal, 1913 ssp. incognita Bíly, 1974

NAVARRA: Garde, 29-V-97, L. Arnáiz leg. (col. BA).

\section{7.- Anthaxia (Melanthaxia) similis Saunders,} 1871

NAVARRA: Garde, 29-V-97, L. Arnáiz leg. (col. BA).

\section{8.- Anthaxia (Melanthaxia) quadripunctata (Linnaeus, 1758)}

GUIPÚZCOA: Zarauz (Teunissen, 1998).

49.- Anthaxia (Melanthaxia) carmen Obenberger, 1912

PALENCIA: Bárcena de Campos, 14-VIII-93, 30-VII-97, 8-VIII-97, P. Bahillo leg. (col. PB) (col. BA) y Calahorra de Boedo, 27-VII-97, 6-VIII-97, P. Bahillo leg. (col. PB) (col. BA).

SORIA: Hinojosa de la Sierra, 11-VII-93, J. Agoiz leg. (col. JLA)

SALAMANCA: El Payo, 4-VII-83, 11-VII-83, J. C. Orella y A. Mateos leg. (col. JLC) y Peñaparda, 9-VII-81, 26-VII-83, H. Santos y J. C. Orella leg. (col. JLC).

LEÓN: Ambasaguas de Curueño, 7-VI-98, L. Arnáiz leg. (col. BA) y Santa María del Condado, 23-VI-98, L. Arnáiz leg. (col. BA).

\section{0.- Anthaxia (Melanthaxia) istriana}

Rosenhauer, 1847

ASTURIAS: Pto. de Pajares (Cobos, 1986).

\section{1.- Anthaxia (Melanthaxia) nigritula} (Ratzeburg, 1837)

ZAMORA: Quiruelas de Vidriales, 16-III-98, P. Bahillo leg. (col. BA).

SALAMANCA: Puebla de Azaba, 1-IV-98, F. Calvo leg. (col. FC); Peñaparda, 11-VII-83, López-Colón leg. (col. JLC) y Robleda, 2-VII-83, A. Justo leg. (col. JLC).

SORIA: Pto. de Piqueras, 4-V-97, F. Calvo leg. (col. FC).

PALENCIA: Dueñas, 5-III-98, 22-III-98, P. Bahillo leg. (col. BA).
Género Melanophila Eschscholtz, 1829 52.- Melanophila acuminata (DeGeer, 1774)

CANTABRIA: Santander alrededores (Cobos, 1986).

ASTURIAS: Portigón, 11-VII-90, M. Sobrino leg. (col. MS).

LEÓN: La Vecilla, 29-VI-84, R. Martínez leg. (col. RM).

SALAMANCA: Robleda, 11-VII-83, A. Justo leg. (col.

JLC) y Peñaparda, 9-VII-81, 25-VI-83, 4-VII-83, H. Santos y J. C. Orella leg. (col. JLC)

53.- Melanophila cuspidata (Klug, 1829) MS).

ORENSE: Razamonde, 19-VIII-91, M. Sobrino leg. (col.

Género Phaenops Lacordaire, 1857

54.- Phaenops cyaneus (Fabricius, 1775)

VIZCAYA: Ochandio, 11-VIII-91, J. Alcorta leg. (col. JA); Urigoiti, 12-VIII-92, I. Alonso leg. (col. IA) y Usabel, 13-VIII92, I. Alonso leg. (col. IA).

CANTABRIA: Potes, 2-VIII-95, L. Arnáiz leg. (col. BA).

NAVARRA: Garde, 30-V-97, L. Arnáiz leg. (col. BA).

PALENCIA: Bárcena de Campos, 7-VI-90, P. Bahillo leg. (col. PB).

SORIA: Pto. de Santa Inés, 24-VII-93, J. Alcorta leg. (col. JA).

LA RIOJA: Villoslada de Cameros, 20-VIII-93, I. Alonso leg. (col. IA)

ORENSE: Razamonde, 22-VII-90, M. Sobrino leg. (col. MS). LEÓN: La Vecilla, 13-VI-85, 25-VI-85, R. Martínez leg. (col. RM) y Portilla, 24-VI-85, R. Martínez leg. (col. RM).

BURGOS: Masa, 21-V-98, P. Bahillo leg. (col. PB).

Género Trachypteris Kirby, 1837

55.- Trachypteris picta (Pallas, 1773) ssp. decostigma (Fabricius, 1787)

ÁlAVA: Baños de Ebro, 27-VII-82, (col. SCNA).

PALENCIA: Bárcena de Campos, 4-VIII-87, P. Bahillo leg. (col. PB).

LEÓN: Valdeteja, 13-VI-85, R. Martínez leg. (col. RM); La Vecilla, 13-VI-85, R. Martínez leg. (col. RM) y Valderas, 30-VI-85, T. Martínez leg. (col. RM).

ZAMORA: Granucillo de Vidriales, 16-VI-91, P. Bahillo leg. (col. PB).

SALAMANCA: Puebla de Azaba, 30-III-97, 27-IV-97, 4VIII-97, F. Calvo leg. (col. FC)

Tribu CHRYSOBOTHRINI Laporte \& Gory, 1837 Género Chrysobothris Eschscholtz, 1829

56.- Chrysobothris affinis (Fabricius, 1794)

ÁLAVA: Pto. de Opakua, 1-VIII-92, P. Bahillo leg. (col. PB); 7-VII-90, 25-VII-90, I. Alonso leg. (col. IA); 8-VIII-87, 31-VII-92, 2-VIII-92, J. Alcorta leg. (col. JA) y Río Bayas, 31VIII-87, I. Alonso leg. (col. IA).

NAVARRA: Garde, 29-V-97, L. Arnáiz leg. (col. BA) y S de Urbasa, 9-VII-94, P. Bahillo leg. (col. PB).

CANTABRIA: Cambarco, 12-VII-86, P. Bercedo leg. (col. BA).

LEÓN: Riaño, 3-VII-85, R. Martínez leg. (col. RM); Valdeón, 6-VII-85, 13-VII-86, R. Martínez leg. (col. RM) y La Vecilla, 6-VIII-86, R. Martínez leg. (col. RM). 
ORENSE: Celanova (Iglesias, 1928) y Orense, 14-VII-90, M. Sobrino leg. (col. MS).

SALAMANCA: Puebla de Azaba, 5-IV-96, F. Calvo leg. (col. BA)

57.- Chrysobothris solieri Laporte \& Gory, 1836 CANTABRIA: Gama, 20-VI-98, P. Bahillo leg. (col. PB). NAVARRA: Garde, 29-V-97, L. Arnáiz leg. (col. BA).

LA RIOJA: Villoslada de Cameros, 20-VIII-93, I. Alonso leg. (col. IA).

BURGOS: Oña, 21-VII-91, L. Arnáiz leg. (col. BA). PALENCIA: Bárcena de Campos, 8-VIII-93, P. Bahillo leg. (col. PB) y Calahorra de Boedo 1-XI-97, P. Bahillo leg. (col. PB)

SALAMANCA: Robleda, 2-VII-83, A. Justo leg. (col. JLC); El Payo, 11-VII-83, 11-VIII-83, A. Mateos leg. (col. JLC) y Peñaparda, 9-VII-81, J. C. Orella leg. (col. JLC).

ZAMORA: Quiruelas de Vidriales, 14-VIII-98, P. Bahillo leg. (col. BA).

Tribu SPHENOPTERINI Lacordaire, 1857

Género Sphenoptera Solier, 1833

Subgénero Sphenoptera Solier, 1833

58.- Sphenoptera (Sphenoptera) barbarica

(Gmelin, 1790)

PALENCIA: Bárcena de Campos, 22-IV-92, 25-VII-97, P. Bahillo leg. (col. PB).

BURGOS: Olmillos de Sasamón, 22-III-97, P. Bahillo leg. (col. PB)

Subgénero Chilostheta Jakovlev, 1889

59.- Sphenoptera (Chilostheta) parvula (Fabricius, 1798)

LEÓN: Astorga (Cobos, 1986).

60.- Sphenoptera (Chilostheta) notha Abeille, 1909

LEÓN: Vegas del Condado, 14-VII-98, 15-VII-98, L. Arnáiz leg. (col. BA).

Subgénero Deudora Jakovlev, 1889 61.- Sphenoptera (Deudora) gemmata (Olivier, 1790)

LEÓN: Valderas, 2-X-84, R. Martínez leg. (col. RM).

Subfamilia AGRILINAE Laporte, 1835

Tribu AGRILINI Laporte, 1835

Género Coroebus Laporte \& Gory, 1839

62.- Coroebus elatus (Fabricius, 1787)

VIZCAYA: Bilbao alrededores (Cobos, 1986). BA).

LEÓN: Vegas del Condado, 17-VII-98, L. Arnáiz leg. (col.

ZAMORA: Ribadelago (Cobos, 1986)

CORUÑA: Betanzos (Cobos, 1986).

63.- Coroebus florentinus (Herbst, 1801)

ÁLAVA: Pto. de Altube, 18-V-96, P. Bahillo leg. (col. PB).

VIZCAYA: Barakaldo, 20-II-96, P. Bahillo leg. (col. PB) y Lejona, 23-VI-81, P. Bahillo leg. (col. PB).
CANTABRIA: Puente Viesgo (Cobos, 1986).

LEÓN: Vegas del Condado, 24-VII-98, L. Arnáiz leg. (col. BA)

64.- Coroebus undatus (Fabricius, 1787)

LA RIOJA: Nieva de Cameros (Cobos, 1986).

SALAMANCA: Puebla de Azaba, 20-VI-97, F. Calvo leg. (col. FC).

Género Meliboeoides Théry, 1942

65.- Meliboeoides amethystinus (Olivier, 1790)

PALENCIA: Torquemada (Teunissen, 1998). MS).

ORENSE: Razamonde, 10-IV-81, M. Sobrino leg. (col.

SALAMANCA: Salamanca, 21-VI-85, T. Martínez leg. (col. RM).

NAVARRA: Pizarrey-Arguedas, 28-VI-96, J. Agoiz leg. (col. BA)

Género Meliboeus Deyrolle, 1864

Subgénero Meliboeus Deyrolle, 1864

66.- Meliboeus (Meliboeus) graminis (Panzer, 1799)

PALENCIA: Bárcena de Campos, 13-VII-86, P. Bahillo leg. (col. PB).

VALLADOLID: Traspiñedo (Cobos, 1986).

67.- Meliboeus (Meliboeus) santolinae (Abeille, 1894)

LEÓN: Vegas del Condado, 11-VII-98, 16-VII-98, L. Arnáiz leg. (col. BA).

Género Nalanda Théry, 1904

68.- Nalanda fulgidicollis (Lucas, [1846])

VIZCAYA: Paules-Las Llanas, 10-VI-91, P. Bahillo leg. (col. PB) y Barakaldo, 17-IV-98, P. Bahillo leg. (col. PB).

CANTABRIA: Potes (Cobos, 1986).

ORENSE: Orense alrededores (Cobos, 1986).

LEÓN: Ambasaguas de Curueño, 7-VI-98, 27-VI-98, L. Arnáiz leg. (col. BA); La Vecilla, 6-VIII-84, R. Martínez leg. (col. RM) y Vegas del Condado, 4-VIII-98, L. Arnáiz leg. (col. BA).

Género Agrilus Curtis, 1825

Subgénero Anambus Thomson, 1864

69.- Agrilus (Anambus) laticornis (Illiger, 1803)

ÁLAVA: Sarria, 19-V-90, I. Alonso leg. (col. PB).

VIZCAYA: Galdames, 29-VII-92, I. Alonso leg. (col. PB) y Güeñes, 10-V-92, P. Bahillo leg. (col. BA).

GUIPÚZCOA: Astigarraga, 30-VI-96, F. Calvo leg. (col. FC).

NAVARRA: Pto. de Arteta-Goñi, 31-V-97, J. Agoiz leg. (col. JLA).

ZAMORA: Ribadelago (Cobos,1986).

LEÓN: Ambasaguas de Curueño, 13-VI-98, L. Arnáiz leg. (col. BA); Vegas del Condado, 13-VI-98, 17-VI-98, L. Arnáiz leg. (col. BA) y Riaño, 8-VII-85, T. Martínez leg. (col. RM).

70.- Agrilus (Anambus) grandiceps Kiesenwetter, 1857 ssp. hemiphanes Marseul, 1865 SORIA: Adradas, 25-VII-97, L. Arnáiz leg. (col. BA). 
71.- Agrilus (Anambus) angustulus (Illiger, 1803) ÁLAVA: Barrio, 28-IV-93, P. Bahillo leg. (col. PB); Pto. de Altube, 27-IV-92, P. Bahillo leg. (col. PB); Pto. de Opakua, 17 III-90, 27-IV-90, P. Bahillo leg. (col. PB) y Abornicano, 24-V92, P. Bahillo leg. (col. PB) (col. BA).

VIZCAYA: Arrancudiaga, 11-VI-90, 22-VI-90, P. Bahillo leg. (col. PB); Barakaldo, 2-VI-90, 2-V-93, 10-V-93, 24-IV-97, P. Bahillo leg. (col. PB); Butrón, 12-V-90, 14-VI-91, P. Bahillo leg. (col. PB); Durango, 20-III-92, P. Bahillo leg. (col. PB); Galdakano, 17-IV-91, 6-V-91, 8-VI-91, P. Bahillo leg. (col. PB); Leioa, 1-VI-93, P. Bahillo leg. (col. PB); Markaida, 14-VI-91, 1VII-91, P. Bahillo leg. (col. PB); Paules-Las Llanas, 3-VI-91, P. Bahillo leg. (col. PB) y Plencia. 10-V-92, P. Bahillo leg. (col. PB).

GUIPÚZCOA: Bedoña, 6-V-93, P. Bahillo leg. (col. PB); Laurgain-Aia, 30-III-92, 27-IV-92, P. Bahillo leg. (col. PB); Motrico (Alto del Calvario), 22-V-94, P. Bahillo leg. (col. PB); Santa Elena-Saturrarán, 27-V-93, P. Bahillo leg. (col. PB); Tolosa, 22-V-94, P. Bahillo leg. (col. PB) y Astigarraga, 12-V97, 18-V-97, 30-V-97, F. Calvo leg. (col. FC) (col. BA).

NAVARRA: Goñi, 31-V-97, J. Agoiz leg. (col. JLA).

CANTABRIA: El Pontarrón (Guriezo), 30-V-92, P. Bahillo leg. (col. BA).

LEÓN: Castro del Condado, 4-VI-98, L. Arnáiz leg. (col. BA); Ambasaguas de Curueño, 6-VI-98, L. Arnáiz leg. (col. BA); Olleros, 20-VI-98, L. Arnáiz leg. (col. BA) y Vegas del Condado, 10-VI-98, L. Arnáiz leg. (col. BA). $\mathrm{PB})$

BURGOS: Barriga de Losa, 10-V-90, P. Bahillo leg. (col

SALAMANCA: Puebla de Azaba, 30-III-97, 12-IV-97, 14IV-97, 27-IV-97, F. Calvo leg. (col. FC) (col. BA).

72.- Agrilus (Anambus) sulcicollis Lacordaire, 1835

ÁLAVA: Murguía, 7-IV-90, 18-IV-98, P. Bahillo leg. (col. PB) (col. BA).

CANTABRIA: Reinosa (Cobos, 1986).

ZAMORA: Ribadelago (Cobos, 1986).

LEÓN: Ambasaguas de Curueño, 7-VI-98, 13-VI-98, L. Arnáiz leg. (col. BA); Vegas del Condado, 20-V-98, L. Arnáiz leg. (col. BA) y Olleros, 20-VI-98, L. Arnáiz leg. (col. BA).

73.- Agrilus (Anambus) hastulifer (Ratzeburg, 1837) ÁLAVA: Sa de Urbasa, 30-VI-96, F. Calvo leg. (col. FC).

ORENSE: Leiro, 16-VII-90, 18-VII-90, M. Sobrino leg. (col. MS) (col. BA) y Razamonde, 2-VIII-94, M. Sobrino leg (col. MS).

LEÓN: Olleros, 20-VI-98, L. Arnáiz leg. (col. BA).

74.- Agrilus (Anambus) olivicolor Kiesenwetter, 1857

CANTABRIA: Beranga, 4-VI-97, L. Arnáiz leg. (col. BA).

75.- Agrilus (Anambus) sinuatus (Olivier, 1790)

ASTURIAS: Las Brañas (Cobos, 1986).

SALAMANCA: Salamanca (Cobos, 1986).

LEÓN: Riaño, 12-VII-98, L. Arnáiz leg. (col. BA).

76.- Agrilus (Anambus) massanensis Schaefer, 1955 BA).

\section{7.- Agrilus (Anambus) biguttatus (Fabricius,} 1777)

ÁLAVA: Pto. Herrera, 2-VII-77, P. Bahillo leg. (col. BA).

CANTABRIA: Cambarco, 12-VII-86, P. Bercedo leg. (col. BA) y Borleña, 7-VII-76, P. Bercedo leg. (col. BA).

ASTURIAS: Mte. Muniellos (Cobos, 1986) y Muniellos, 15-VIII-85, R. Martínez leg. (col. RM).

LA RIOJA: Panzaros (debe tratarse de Panzares) (Teunissen, 1998).

LEÓN: Vegas del Condado, 7-VII-98, L. Arnáiz leg. (col. BA); Ambasaguas de Curueño, 28-VI-98, L. Arnáiz leg. (col. BA) y Lugán, 16-VI-98, L. Arnáiz leg. (col. BA).

SALAMANCA: Salamanca alrededores (Cobos, 1986) y Béjar (Cobos, 1986).

ORENSE: Orense alrededores (Cobos, 1986) y Razamonde, 9-IV-81, M. Sobrino leg. (col. MS).

78.- Agrilus (Anambus) graminis Laporte \& Gory, 1839

ÁLAVA: Sa de Urbasa, 30-VI-96, F. Calvo leg. (col. FC).

LEÓN: Vegas del Condado, 18-VII-98, L. Arnáiz leg. (col. BA) y Ambasaguas de Curueño, 19-VII-98, L. Arnáiz leg. (col. BA).

SALAMANCA: Puebla de Azaba, 14-IV-96, F. Calvo leg. (col. FC).

ORENSE: Pto. Estivadas (Teunissen, 1998).

79.- Agrilus (Anambus) beauprei Théry, 1928 ssp. mourguesi Schaefer, 1954

NAVARRA: Soto de la Remonta (Tudela), 27-V-97, 28-V97, J. Agoiz leg. (col. BA).

LEÓN: Vegas del Condado, 5-VI-98, L. Arnáiz leg. (col. BA).

80.-Agrilus (Anambus) curtulus Mulsant \& Rey, 1863

LEÓN: Ambasaguas de Curueño, 19-VII-98, L. Arnáiz leg. (col. BA) y Vegas del Condado, 18-VII-98, L. Arnáiz leg. (col. BA).

PALENCIA: Bárcena de Campos, 30-VII-97, P. Bahillo leg. (col. PB).

81.- Agrilus (Anambus) cyanescens (Ratzeburg, 1837)

SALAMANCA: Béjar (Cobos, 1986).

82.- Agrilus (Anambus) albogularis Gory, 1841 ssp. artemisiae Brisout, 1863

LEÓN: Vegas del Condado, 16-VII-98, 17-VII-98, L. Arnáiz leg. (col. BA).

\section{3.- Agrilus (Anambus) pratensis (Ratzeburg,} 1839) ssp. pratensis

LEON: Vegas del Condado, 11-VII-98, 13-VII-98, 14-VII98, L. Arnáiz leg. (col. BA).

Subgénero Agrilus Curtis, 1825

84.- Agrilus (Agrilus) viridis (Linnaeus, 1758)

ÁLAVA: Pto. de Altube, 31-I-98, P. Bahillo leg. (col. PB). 
LA RIOJA: Nieva de Cameros (Cobos, 1986).

LEÓN: Valdeón, 7-VII-85, T. Martínez leg. (col. RM) y Vegas del Condado, 15-VII-98, L. Arnáiz leg. (col. BA).

85.- Agrilus (Agrilus) hyperici (Creutzer, 1799) CANTABRIA: Potes (Cobos, 1986).

LEÓN: Vegas del Condado, 18-VII-98, L. Arnáiz leg. (col. BA).

PONTEVEDRA: Pontevedra alrededores (Cobos, 1986). ORENSE: Barco de Valdeorras (Cobos, 1986).

SALAMANCA: Sequeros (Cobos, 1986) y Monsagro (Cobos, 1986).

PALENCIA: Bárcena de Campos, 25-VII-97, P. Bahillo leg. (col. PB).

86.- Agrilus (Agrilus) antiquus Mulsant \& Rey, 1863 ssp. hispanicus Obenberger, 1913

ASTURIAS: Asturias (Cobos, 1986).

87.- Agrilus (Agrilus) cinctus (Olivier, 1790)

LUGO: Sober (Cobos, 1986) y S de Ancares (Cobos, 1986). ORENSE: Castro Caldelas (Cobos, 1986).

LEÓN: Riaño (Cobos, 1986), Villablino (Cobos, 1986) y Ambasaguas de Curueño, 27-VI-98, 28-VI-98, 8-VII-98, L. Arnáiz leg. (col. BA).

SALAMANCA: Candelaria (Cobos, 1986) y Candelario, 20-VII-92, M. Sobrino leg. (col. MS).

88.- Agrilus (Agrilus) moriscus Obenberger, 1913 LA RIOJA: Calahorra (Cobos, 1986).

CANTABRIA: Pto. de San Glorio, 4-IX-96, L. Arnáiz leg. (col. Curletti).

LEÓN: Cospedal, 1-VII-98, 3-VII-98, L. Arnáiz leg. (col. BA)

89.- Agrilus (Agrilus) uhagoni Abeille, 1897

ASTURIAS: Pto. de Pajares (Cobos, 1986).

SALAMANCA: Monsagro (Cobos, 1986).

LEÓN: Ambasaguas de Curueño, 9-VII-98, L. Arnáiz leg. (col. BA)

90.- Agrilus (Agrilus) pulvereus Abeille, 1895

LA RIOJA: Calahorra (Cobos, 1986) y Pradejón, 27-VII95, 26-VII-98, L. Arnáiz leg. (col. BA).

NAVARRA: Las Norias (Tudela), 30-VI-97, J. Agoiz leg. (col. JLA) (col. BA).

91.- Agrilus (Agrilus) salicis Frivaldszky, 1877 VALLADOLID: Olmedo (Cobos, 1986).

92.- Agrilus (Agrilus) suvorovi Obenberger, 1935 ssp. populneus Schaefer, 1946

LEÓN: Valdeón, 6-VII-85, T. Martínez leg. (col. BA).

Tribu TRACHYINI Laporte, 1835

Género Aphanisticus Latreille, 1810

93.-Aphanisticus elongatus Villa \& Villa, 1835 ssp. elongatus

LEÓN: Alto de Picones, 8-V-98, L. Arnáiz leg. (col. BA).
94.- Aphanisticus emarginatus (Olivier, 1790)

"Prácticamente todo el país, incluida Galicia (Champión)" (sic) (Cobos, 1986).

95.- Aphanisticus angustatus Lucas, [1846]

LA RIOJA: Rincón de Soto (Cobos, 1986).

96.- Aphanisticus pusillus (Olivier, 1790) GUIPÚZCOA: Zumaya (Cobos, 1986).

PONTEVEDRA: Pontevedra alrededores (Cobos, 1986).

LEÓN: León alrededores (Cobos, 1986).

Género Habroloma Thomson, 1864

97.- Habroloma triangulare (Lacordaire, 1835)

VALLADOLID: Olmedo (Cobos, 1986).

Género Trachys Fabricius, 1801

98.- Trachys coruscus (Ponza, 1805)

VIZCAYA: Zarátamo, 2-VII-87, P. Bahillo leg. (col. BA) y Barakaldo, 31-III-90, P. Bahillo leg. (col. PB).

LEÓN: Corullón, 8-VII-85, T. Martínez leg. (col. RM).

CANTABRIA: Argoños, 9-V-98, P. Bahillo leg. (col. PB) (col. BA).

Presente en toda la Península Ibérica (Cobos, 1986).

99.- Trachys reflexus Gené, 1839

LA RIOJA: Villarejo (Cobos, 1986).

LEÓN: Vegas del Condado, 17-VII-98, 7-VIII-98, L. Arnáiz leg. (col. BA).

100.- Trachys minutus (Linnaeus, 1758)

ASTURIAS: Oviedo alrededores y Mte. Muniellos, Moal (Cobos, 1986).

101.- Trachys troglodytes Gyllenhal, 1817 ssp. compressus Abeille, 1891

CANTABRIA: Santander alrededores (Cobos, 1986) y Esles, VI-78, P. Bercedo leg. (col. BA).

102.- Trachys scrobiculatus Kiesenwetter, 1857

"Probablemente todo el territorio entre el nivel del mar y cerca de los $1.000 \mathrm{~m} / \mathrm{a} "($ sic) (Cobos, 1986).

Tribu CYLINDROMORPHINI Portevin, 1931

Género Cylindromorphus Kiesenwetter, 1857

Cylindromorphus parallelus Fairmaire, 1859

PALENCIA: Palencia (Cobos, 1986).

\section{Correcciones a la nomenclatura}

En el presente trabajo se hacen las siguientes precisiones sobre aspectos nomenclaturales:

1.- Las especies del género Acmaeodera Eschscholtz, 1829, A. nigellata Abeille, 1904 y A. bipunctata (Olivier, 1790) se incluyen en el subgénero Palaeotethya Volkovitsh, 1979 (en el 
que también queda incluida la especie $A$. rubromaculata Lucas, 1844 ssp. segurensis Escalera, 1904 y A. rubromaculata Lucas, 1844 ssp. malacensis Cobos, 1954) (Volkovitsh, 1979), y no en el subgénero Loepotethya Volkovitsh, 1979 citado erroneamente como ibérico (Cobos, 1986).

2.- Se cita Anthaxia (Anthaxia) thalassophila Abeille, 1900 ssp. iberica Cobos, 1986, en lugar de Anthaxia (Anthaxia) podolica Mannerheim, 1837 ssp. iberica Cobos, 1986, siguiendo el criterio de Schaeffer (1949) en el que considera las citas de España y Portugal como A. thalassophila Abeille, 1900 al igual que Bíly (1997).

3.- Asimismo se considera Anthaxia (Melanthaxia) carmen Obenberger, 1912 como especie siguiendo a Schaefer (1949) y Bíly (1997) y no como ssp. de Anthaxia (Melanthaxia) corsica Reiche, 1861 (Cobos, 1986).

4.- En este trabajo se considera el género Palmar Schaefer, 1949. Palmar fue creado como subgénero de Lampra Dejean, 1833; Hellrigl (1972) lo elevó al rango de género. Lampra es en la actualidad sinónimo de Palmar. Cobos (1986) incluía Palmar como subgénero de Ovalisia Kerremans, 1900 pero éste último es un género tropical (islas Salomón).

5.- Cobos (1986) engloba en el subgénero Anthaxia Eschscholtz, 1829 lo que en la actualidad son dos subgéneros: subgénero Anthaxia Eschscholtz, 1829 y subgénero Melanthaxia Richter, 1949 (Bíly, 1982). Las especies mencionadas en este trabajo que pertenecen al subgénero Melanthaxia Richter, 1949 son:

- Anthaxia (Melanthaxia) godeti Laporte \& Gory, 1839

- Anthaxia (Melanthaxia) sepulchralis (Fabricius, 1801)

- Anthaxia (Melanthaxia) nigrojubata Roubal, 1913 ssp. incognita Bíly, 1974

- Anthaxia (Melanthaxia) similis Saunders, 1871

- Anthaxia (Melanthaxia) quadripunctata (Linnaeus, 1758)

- Anthaxia (Melanthaxia) carmen Obenberger, 1912

- Anthaxia (Melanthaxia) istriana Rosenhauer, 1847

- Anthaxia (Melanthaxia) nigritula (Ratzeburg, 1837)

El resto de especies ibéricas que forman este subgénero son las siguientes:

- Anthaxia (Melanthaxia) castiliana Obenberger, 1914
- Anthaxia (Melanthaxia) espanoli Cobos, 1954

- Anthaxia (Melanthaxia) rugicollis Lucas, [1846]

- Anthaxia (Melanthaxia) segurensis, Obenberger, 1924

6.- Se hacen correcciones en el nombre y año de descripción de los siguientes táxones:

- Acmaeodera (Acmaeodera) pulchra (Fabricius, 1792)

- Acmaeodera (Acmaeotethya) crinita Gory, 1840 ssp. melanosoma Lucas, 1844

- Acmaeoderella (Euacmaeoderella) cyanipennis (Lucas, [1846]) ssp. hispana (Abeille, 1900)

- Capnodis tenebrionis (Linnaeus, 1761)

- Cyphosoma lausoniae (Chevrolat, 1838)

- Palmar festiva (Linnaeus, 1767)

- Anthaxia (Haplanthaxia) parallela Laporte \& Gory, 1839

- Anthaxia (Anthaxia) funerula (Illiger, 1803) ssp. funerula

- Anthaxia (Melanthaxia) nigritula (Ratzeburg, 1837)

- Anthaxia (Melanthaxia) espanoli Cobos, 1954

- Anthaxia (Melanthaxia) rugicollis Lucas, [1846]

- Phaenops cyaneus (Fabricius, 1775)

- Trachypteris picta (Pallas, 1773) ssp. decostigma (Fabricius, 1787)

- Chrysobothris solieri Laporte \& Gory, 1836

- Sphenoptera (Sphenoptera) barbarica (Gmelin, 1790)

- Meliboeus (Meliboeus) graminis (Panzer, 1799)

- Nalanda fulgidicollis (Lucas, [1846])

- Agrilus (Anambus) hastulifer (Ratzeburg, 1837)

- Agrilus (Anambus) cyanescens (Ratzeburg, 1837)

- Agrilus (Anambus) pratensis (Ratzeburg, 1839) ssp. pratensis

- Agrilus (Agrilus) suvorovi Obenberger, 1935 ssp. populneus Schaefer, 1946

- Aphanisticus elongatus Villa \& Villa, 1835 ssp. elongatus

- Aphanisticus angustatus Lucas, [1846]

- Habroloma triangulare (Lacordaire, 1835)

- Trachys coruscus (Ponza, 1805)

- Trachys reflexus Gené, 1839

- Trachys troglodytes Gyllenhal, 1817 ssp. compressus Abeille, 1891

- Trachys scrobiculatus Kiesenwetter, 1857 


\section{Discusión}

Entre los táxones citados en el presente trabajo merecen ser destacados:

- Agrilus (Anambus) pratensis pratensis (Ratzeburg, 1839) citado por primera vez para la Península Ibérica del Pirineo ilerdense (Arnáiz Ruiz, 1998). La aparición de esta especie en Vegas del Condado (León) es la segunda cita conocida. Dada la gran similitud con Agrilus (Anambus) massanensis Schaefer, 1955 puede hacer pensar que en muchas colecciones estas especies estén confundidas y por eso las pocas citas conocidas. Los ejemplares han sido capturados sobre Populus x canadensis Moench, en plantones jóvenes: Vegas del Condado, VII-1998, 35 ejs. ( $\sigma^{\nearrow} \sigma^{7}$, 우 ㅇ ), L. Arnáiz leg. (col. BA). Todo esto hace suponer la presencia de esta especie al menos en el tercio septentrional peninsular.

- Sphenoptera (Chilostheta) notha Abeille, 1909 considerado un endemismo ibérico central (Cobos, 1986). Se conocía de varias localidades de la provincia de Madrid (Cobos, 1986; Compte, 1988) y de Albalate de Zorita en la provincia de Guadalajara (Arnáiz Ruiz, 1996b). La cita aportada en el presente trabajo de León: Vegas del Condado, VII-1998, 25 ejs. ( $\sigma^{\top} \sigma^{\top}$, 우 우) L. Arnáiz leg. (col. BA), puede considerarse muy importante, ya que la distancia geográfica con las dos provincias antes mencionadas es considerable y por lo tanto puede suponerse una distribución mucho más amplia de la especie.

- Agrilus (Anambus) grandiceps hemiphanes Marseul, 1865 especie considerada como rara y citada tan solo de las provincias de Gerona, Barcelona, Cuenca y Teruel (Cobos, 1986). Además de la cita aportada en el presente trabajo de Soria: Adradas, 2 ejs. 우 웅 25-VII-97, L. Arnáiz leg. (col. BA) se considera necesario dar nuevas localizaciones de otras provincias así como confirmar nuevos enclaves de Barcelona y Cuenca. Albalate de Zorita (Guadalajara) $10^{7}$ y 1 ㅇ , 30-VI-96. Campo Real (Madrid) 1 우, 15-VI95. Pto. de Cabrejas (Cuenca) $1 \sigma^{7}$ y 1 우, 8-VII95; todas estas citas están legitimadas por el autor de esta nota (col. BA). La Panadella (Barcelona) 1 o', 19-VI-97, J. L. Agoiz leg. (col. BA). Todos los ejemplares fueron recolectados en pequeñas plantas de Quercus ilex L. subsp. ballota (Desf.) Samp, y Quercus coccifera L.

- Agrilus (Anambus) curtulus Mulsant \& Rey, 1863 al igual que la especie anterior se la considera rara, citada tan solo de Madrid (Cobos, 1986). Las citas aquí aportadas de León: Ambasaguas de
Curueño 6 ejs. ( $\sigma^{7} \sigma^{\pi}$, 우 우), 19-VII-98 y Vegas del Condado 8 ejs. ( $\sigma^{\top} \sigma^{\top}$, ㅇ ㅇ ), 18-VII-98, L. Arnáiz leg. (col. BA); junto con la cita de Palencia: Bárcena de Campos 1 오, 30-VII-97, P. Bahillo leg. (col. PB); y otra de la provincia de Segovia, de San Rafael (El Espinar) 1 ণ゙, 8-VII-89, J. I. López-Colón leg. (col. BA) amplían su distribución. Los ejemplares capturados en León fueron recolectados sobre Quercus pyrenaica Willd.

- Agrilus (Agrilus) suvorovi populneus Schaefer, 1946 citado de la Casa de Campo y Villaviciosa de Odón en la provincia de Madrid (Cobos, 1986). Otras citas de Madrid: Villar del Olmo,

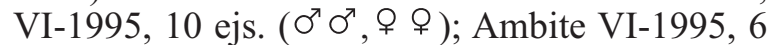
ejs. ( $\sigma^{7}$, , $\odot$ ㅇ ), L. Arnáiz leg. (col. BA). En la provincia de León se ha localizado en Valdeón 1 ㅇ , 6-VII-85, T. Martinez leg. (col. BA).

También resaltar que existe un número de especies perfectamente asentadas en todo el territorio nacional, pudiéndose relacionar entre otras:

- Acmaeoderella (Omphalothorax) adspersula (Illiger, 1803)

- Acmaeoderella (Carininota) flavofasciata flavofasciata (Piller \& Mitterpacher, 1783)

- Ptosima flavoguttata (Illiger, 1803)

- Anthaxia (Haplanthaxia) umbellatarum (Fabricius, 1787)

- Anthaxia (Haplanthaxia) scutellaris Gené, 1839

- Anthaxia (Haplanthaxia) millefolii polychloros Abeille, 1894

- Anthaxia (Anthaxia) cyanescens bedeli Abeille, 1893

- Anthaxia (Anthaxia) funerula funerula (Illiger, 1803)

- Anthaxia (Anthaxia) manca (Linnaeus, 1767)

- Anthaxia (Melanthaxia) carmen Obenberger, 1912

- Anthaxia (Melanthaxia) nigritula (Ratzeburg, 1837)

- Anthaxia (Cratomerus) hungarica (Scopoli, 1772)

- Buprestis (Buprestis) novemmaculata Linnaeus, 1767

- Meliboeoides amethystinus (Olivier, 1790)

- Nalanda fulgidicollis (Lucas, [1846])

- Coroebus elatus (Fabricius, 1787)

- Agrilus (Anambus) biguttatus (Fabricius, 1777)

- Agrilus (Anambus) angustulus (Illiger, 1803)

- Agrilus (Agrilus) hyperici (Creutzer, 1799)

- Aphanisticus emarginatus (Olivier, 1790)

- Trachys coruscus (Ponza, 1805)

- Trachys scrobiculatus Kiesenwetter, 1857 
- Anthaxia (Anthaxia) hypomelaena (Illiger, 1803) citada del levante, centro y sur (Cobos, 1986), que junto a las citas aquí aportadas, a las que se debe añadir otra de Barcelona: La Panadella, 4VI-96, J. L. Agoiz leg. (col. BA), hacen suponer tambien una distribución por todo el territorio nacional.

- Capnodis tenebrionis (Linnaeus, 1761) presente según Cobos (1986) en: "todo el país salvo el noroeste (Galicia, León, cornisa cantábrica), gradualmente enrarecido hacia el Norte" ( citas de Vizcaya: Bazan, 1-X-96, R. F. de Gamboa leg. (col. RFG) y de Palencia: Bárcena de Campos, 3-VIII-95, P. Bahillo leg. (col. PB), también confirman una distribución más amplia.

- Acmaeodera (Acmaeodera) pilosellae (Bonelli, 1812) que si bien no se trata de una especie rara, sin embargo está muy poco representada en las colecciones entomológicas. Especie propia del mediodía y levante (Cobos, 1986). Distribución ampliada a las provincias de Zaragoza y Huesca (Murria, 1994). Las citas aportadas en el presente trabajo de Salamanca: Puebla de Azaba, 30-III97, 1 \% , F. Calvo leg. (col. FC); León: Ambasaguas de Curueño, 7-VI-98, 7 ejs. ( $0^{\prime \prime} 0^{\prime \prime}$, O $\left.\bigcirc\right)$, L. Arnáiz leg. (col. BA); así como una cita de Granada: Sierra Nevada, 24-V-91, 2 $0^{x} 0^{x}$ y 2 \& $\odot$, L. Arnaiz leg. (col. BA) que, con seguridad, constituye una primera localización de la especie en la zona sur de la península, hacen suponer una presencia de este taxon por todo el territorio nacional.

- Buprestis (Buprestis) rustica Linnaeus, 1758 especie limitada, según Cobos (1986), a los valles pirenaicos. Otras citas conocidas: Huesca (varias localidades) y Teruel (Murria, 1994, 1998). La cita aquí aportada de Soria: Pto. de Santa Inés, 18-VIII-94, 1 o $^{7}$, I. Alonso leg. (col. Bellamy), hacen pensar en una distribución mucho más amplia de la especie.

Por último se da una pequeña relación porcentual de las especies, relación que irá en aumento con estudios más profundos de la zona motivo de este trabajo.

Cobos (1986) relaciona un total, entre especies y subespecies, de 178 táxones a nivel peninsular, de los cuales 29 son especies endémicas. A estos táxones deben añadirse: Chalcophora mariana (Linnaeus, 1758) ssp. intermedia (Rey, 1890) (Murria y Murria, 1998), Anthaxia (Haplanthaxia) lusitanica Obenberger, 1943 (Arnáiz Ruiz \& Coello, 1997), Agrilus (Anambus) pratensis (Ratzeburg, 1839) ssp. pratensis (Arnáiz Ruiz, 1998) y Agrilus (Uragrilus) ater (Linnaeus, 1767)
(Murria y Murria, 1996). De estos táxones Anthaxia (Haplanthaxia) lusitanica Obenberger, 1943 sólo se conoce de la Península Ibérica, por lo que se puede considerar un endemismo ibérico.

De la zona de Galicia y cornisa cantábrica Cobos (1986) cita un total de 20 táxones lo que representa el $10,9 \%$ que se puede considerar muy bajo. Aquí se citan para esta misma zona un total de 46 táxones lo que supone un 25,3\%.

Del total de los táxones del presente trabajo, 62 corresponden al País Vasco y provincias limítrofes lo que representa el $34,1 \%$.

Para la comunidad de Castilla-León (con la excepción de las provincias de Segovia y Ávila) se citan un total de 81 táxones lo que supone un $44,5 \%$.

Cobos (1986) cita como endémicas - especies y subespecies - 29 táxones que incluyendo Anthaxia (Haplantaxia) lusitanica Obenberger, 1943 hacen un total de 30 endemismos ibéricos. En el presente trabajo aparecen 10 endemismos lo que representa el $33,3 \%$.

El total de los táxones citados es de 103 lo que representa el $56,6 \%$. Se puede considerar un porcentaje bastante alto considerando el carácter termófilo de esta familia y la climatología de la zona de estudio, con unas temperaturas, en la época de avivamiento de estos insectos, muy inferiores a zonas como el levante, meseta central y mitad meridional penínsular.

\section{AGRADECIMIENTOS}

Al Dr. Miguel Ángel Alonso Zarazaga por su ayuda en las correcciones de la nomenclatura.

A todas las personas mencionadas al principio del presente trabajo, por permitir el estudio de sus colecciones, aportar desinteresadamente datos de captura, así como el envío de material para su estudio, mi agradecimiento más sincero.

A mi marido Pablo Bercedo por su paciencia, ayuda y acertados consejos.

\section{Referencias}

ARNÁIz RuIZ, L., 1996a. Nuevos aportes a la corología de Buprestidae, para la Comunidad Autónoma de Cantabria (Coleoptera, Buprestidae). Bol. Soc. Entomol. Aragon., 13: 63.

ARNÁIZ RUIZ, L., 1996b. Nuevas aportaciones corológicas de Coleoptera Buprestidae para la Península Ibérica. Bol. Soc. Entomol. Aragon., 14: 64.

ArNÁiz Ruiz, L., 1997. Nuevas aportaciones corológicas de Coleoptera Buprestidae para la Península Ibérica. (2 $2^{\mathrm{a}}$ parte). Bol. Soc. Entomol. Aragon., 19: 56. 
Arnáiz RuIz, L., 1998. Primera cita para la Península Ibérica de Agrilus (Anambus) pratensis pratensis Ratzeburg, 1837 (Col., Buprestidae). Bol. Soc. Entomol. Aragon., 21: 4.

Arnáiz Ruiz, L. y Coello, P., 1997. Rehabilitación de Anthaxia (Haplanthaxia) lusitanica Obenberger, 1943 y descripción del macho de dicha especie (Coleoptera: Buprestidae). Bol. Soc. Entomol. Aragon., 18: 7-9.

Bíly, S., 1982. The Buprestidae (Coleoptera) of Fennoscandia and Denmark. Fauna Entomol. Scand., 10: 1110.

Bíly, S., 1997. World Catalogue of the Genus Anthaxia Eschscholtz, 1829 (Coleoptera: Buprestidae). Folia Heyrovsk. Supl., 2: 3-190.

Cobos, A., 1986. Fauna Ibérica de Coleópteros Buprestidae. C.S.I.C. Madrid. 364 pp.

Compte, A., 1988. Bupréstidos de la Provincia de Madrid (Coleópteros). Actas III Congr. Ibér. Entomol:: 323-332.

Hellrigl, K. G., 1972. Revision der westpaläarktischen Arten der Prachtkäfergattung Lampra Lac. (Coleoptera, Buprestidae). Ann. Naturhist. Mus. Wien, 76: 649-708.

Iglesias, L., 1928. Notas Entomoloxicas. Insectos de Galiza I. Coleópteros. Rev. Nós, 53: 89-94.

Murria, F., 1994. Insecta: Coleoptera 6. Familia Buprestidae. Cat. Entomofauna Aragon., 3: 1-15.

MurriA, F., 1998. Datos sobre Coleoptera Buprestidae aragoneses. Cat. Entomofauna Aragon., 17: 19-20.

Murria, F. y Murria, A., 1996. Una nueva especie de Coleoptera Buprestidae para la Península Ibérica. Bol. Soc. Entomol. Aragon., 14: 64.
Murria, F. y Murria, A., 1998. Capturas accidentales de bupréstidos en Aragón (Coleoptera, Buprestidae). Bol. Soc. Entomol. Aragon., 22: 30.

Schaefer, L., 1949. Les Buprestides de France. Misc. Ent. Le Moult. París. 511 pp.

Teunissen, A., 1998. Coleópteros de la Península Ibérica de la colección A. Teunissen (Holanda). I. Familias: Oedemeridae, Buprestidae, Vesperidae, Lucanidae y Rhipiphoridae. Cat. Entomofauna Aragon, 17: 2122.

Volkovitsh, M. G., 1979. A review of Paleartic groups of the Tribe Acmaeoderini (Coleoptera, Buprestidae). Entomol. Obozr., 58(2): 333-354 
Apéndice 1. - Mapa de España mostrando las provincias que abarca el estudio.

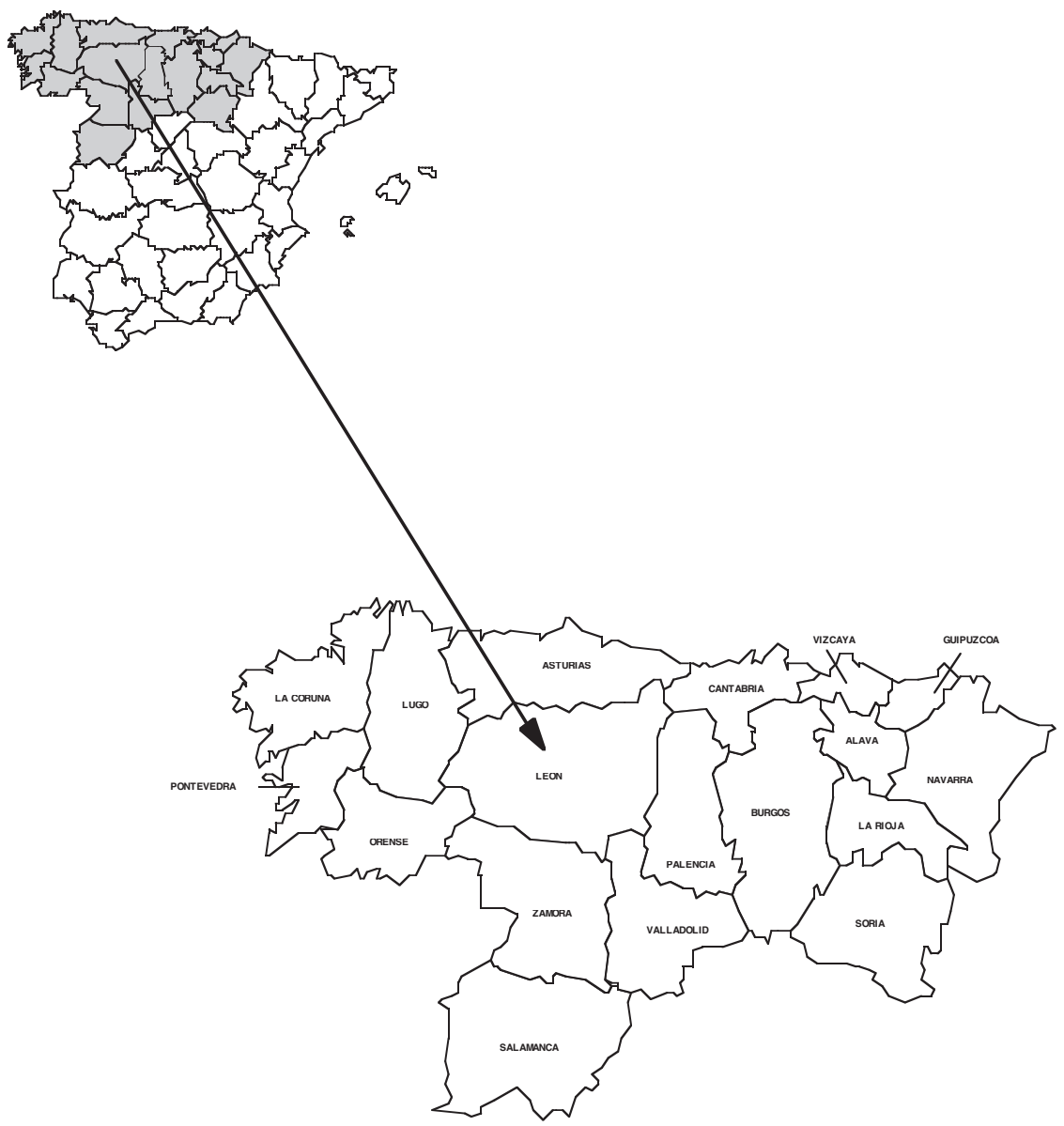

